\title{
TENSOR PRODUCTS OF PARTIAL ALGEBRAS
}

\author{
M. MONSERRAT, F. ROSSELLó' ${ }^{\prime}$ AND I. TORmENS
}

To Pere Menal, in memoniam

\begin{abstract}
In this paper we introduco the tensor product of partial algebras w.r.b. a cuasi-primitive class of partial algclmas, and we prove some of its main propertics. This construction generalizes the well-known tensor product of total algebras w.r.t. varioties.
\end{abstract}

In [Fra], the tensor product on a variety is introduced, and its main properties are studied in [Gal].

The main goal of this papel is to show the existence of the tensor product of two partial algebras with respect to a class of partial algebras (of the sanc type) closed under isomorphic images, direct products and closed subalgebras. Then, the aforementioned tensor product on a variety becomes a special case of our construction.

Moreover, we prove that one of the main properties of the tensor product on a variety ("Thm. 3 in [Gal]) still holds for tensor products of partial algebras. Finally, we use some general results from [BMRRT] to give explicit computations of the tensor product, w.r.t. a class $M$ when such $M$ is either the class of all weak subalgebras of algebras in a varicty $K$, or the class of all partial algebras which satisfy weakly the equational theory of a variety $K$.

Our interest in tensor products (for partial algebras) stems from the study of the behaviour under it of some constructions and properties for temal algebras (algebraic models for systems of inference mules on incomplete data; see [BR] for a recent survey). We will report on this topic clsewhere.

\footnotetext{
TPartially supported by UPC IPH9014.
} 


\section{Preliminaries}

In this section we gather some definitions and results which shall be used in this paper. For any other terminology not introduced in this section we refer the reader to [Bur] (for partial algebras) and [BuS] (for total algebras).

Definition 1. Lct $M$ be a non-empty class of partial algebras of the same type.

a) $M$ is quasi-primitive iff it is closed under isomorphic images, closed subalgebras and direct products.

b) $M$ is weakly quasi-primitive iff it is closed under isomorphic images, weak subalgebras and direct products.

For instance, if $K$ is a variety of total algebras, then $K$ is quasiprimitive, whilc the classes

$W(K)=\{\mathbf{A} \mid \mathbf{A}$ is isomorphic to a weak subalgebra of an algebra in $K\}$

and

$$
E(K)=\left\{\mathrm{A} \mid \mathrm{A} \models_{w} \mathrm{Eq}_{\mathcal{X}}(K)\right\}
$$

(where $F_{w}$ denotes "weak satisfaction" in the sense of, for instance, $[\mathrm{Ru}]$, and $\mathrm{Eq}_{\chi}(K)$ denotes the equational theory of $K$ over a (countably infinite) set of variables $\mathcal{X}$ ) are weakly quasi-primitive. We shall call them the weak subalgebras class and the weak equational class of $K$, respectively.

The following result, due to J. Schmidt. (see [Sch]) states that quasiprimitive classes admit "universal solutions".

Theorem 1. Let $\mathbf{A}$ be an arbitrary partial algebra and $M$ a quasiprimitive class of algebras of the same lype. Then there exists an algebra $\mathrm{B} \in M$ and a homomorphism $h: \mathrm{A} \rightarrow \mathrm{B}$ satisfying the following universal property:

for any $\mathrm{C} \in M$ and any homomorphism $g: \mathbf{A} \rightarrow \mathbf{C}$ there exists a unique homomorphism $\hat{g}: \mathbf{B} \rightarrow \mathrm{C}$ such that $g=\hat{g} h$ :

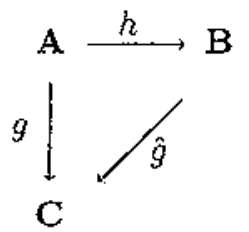


It follows that such a homomorphism $h: \mathbf{A} \rightarrow \mathbf{B}$ is unique up to isomorphism.

Definition 2. A homomorphism $h: \mathbf{A} \rightarrow \mathbf{B}$, with $\mathbf{B} \in M$, satisfying the universal property of the last, theorem is called an $M$-reflection of A.

As a byproduct of some resuits in [BMRRT] it turns out that such an $M$-reflection can be explicitly computed for $M$ a variety, the weak subalgebras class of a varicty and the weak equational class of a variety, respectively. For the convenience of the reader, we recall here (without proofs) the corresponding results.

Let us fix henceforth a comtably infinite set of variables $\mathcal{X}$, and let $T_{\mathcal{F}}(\mathcal{X})$ be the set of terms of upe $\mathcal{F}$ over $\mathcal{X}$.

Theorem 2. Let $\mathbf{A}=\left(A,\left(f^{\mathbf{A}}\right)_{f \in F^{*}}\right)$ be a partial algebra of type $\mathcal{F}=$ $(F, \eta)$. Let $K$ be a variety of tolal algebras of type $\mathcal{F}$ and let $\mathcal{E}=E q_{\mathcal{X}}(K)$ be the equational theory of $K$ w.r.t. $\mathcal{X}$. Let $M=E(K)$ be the weak equational class of $K$.

Consider the congruence on $\mathbf{A}$

$$
\theta_{e}^{\mathbf{A}, M}=\bigcup_{i \geq 0} \theta_{i}^{\mathbf{A}, M}
$$

where $\theta_{i}^{\mathbf{A}}{ }^{M}, i \geq 0$, are defined recurrently as follows: $\theta_{0}^{\mathrm{A}, M}=i d_{A}$ and

$$
\begin{aligned}
\theta_{i+1}^{\mathbf{A}, M}= & \left\{(a, b) \in A^{2} \mid \exists m \geq 1,\right. \\
& \exists\left(a_{1}, c_{1}\right), \ldots,\left(a_{m}, c_{m}\right),\left(c_{1}, b_{1}\right), \ldots,\left(c_{m n}, b_{m}\right) \in \theta_{i}^{\mathrm{A}, M}, \\
& \exists \mathrm{p}_{1}, \mathrm{p}_{2}, \mathrm{q}_{1}, \mathrm{q}_{2} \in T_{\mathcal{F}}(\mathcal{X}) \text { such that } \mathrm{p}_{1} \approx \mathrm{p}_{2}, \mathrm{q}_{1} \approx \mathrm{q}_{2} \in \mathcal{E}, \\
& \left(a_{1}, \ldots, a_{m n}\right) \in \operatorname{dom} \mathrm{p}_{1}^{\mathbf{A}} \text { and } \mathrm{p}_{1}^{\mathbf{A}}\left(a_{1}, \ldots, a_{m}\right)=a_{1}, \\
& \left(b_{1}, \ldots, b_{m}\right) \in \operatorname{dom} \mathrm{q}_{1}^{\mathbf{A}} \text { and } \mathrm{q}_{1}^{\mathbf{A}}\left(b_{1}, \ldots, b_{m}\right)=b, \\
& \left(c_{1}, \ldots, c_{7 n}\right) \in \operatorname{dom} \mathrm{p}_{2}^{\mathbf{A}} \cap \operatorname{dom} \mathrm{q}_{2}^{\mathbf{A}} \text { and } \\
& \left.\mathrm{p}_{2}^{\mathbf{A}}\left(c_{1}, \ldots, c_{m n}\right)=\mathrm{q}_{2}^{\mathbf{A}}\left(c_{1}, \ldots, c_{m}\right)\right\}
\end{aligned}
$$

Then the nalural homomorphism nat $\theta_{\theta_{e}}: \mathbf{A} \rightarrow \mathbf{A} / \theta_{c}^{\mathbf{A}}$ as is an $M$-reflection of $\mathbf{A}$.

Theorem 3. Let $\mathrm{A}=\left(A,\left(f^{\mathrm{A}}\right)_{f \in F}\right), K$ and $\mathcal{E}$ be as in the previous theorem. Let $M=W(K)$ be the weak subalgebras class of $K$. Let $i$ : $\mathbf{A} \rightarrow F(\mathbf{A})$ be the free completion of $\mathbf{A}$ (see [Bur 1.5.4]) and let $F(A)$ be the carrier of $F(\mathbf{A})$. 
Consider the congruence on $F(\mathbf{A})$

$$
\theta_{c}^{F(\mathbf{A}), K}=\bigcup_{i \geq 0} \theta_{i}^{F(\mathbf{A}), K}
$$

with $\theta_{i}^{F(\mathbf{A}), K}, i \geq 0$, defined recurrently as follows: $\theta_{0}^{F(\mathbf{A}), K}=i d_{A}$ and

$$
\begin{aligned}
\theta_{i+1}^{F(\mathbf{A}), K}= & \left\{(a, b) \in F(A)^{2} \mid \exists m \geq 1,\right. \\
& \exists\left(a_{1}, c_{1}\right), \ldots,\left(a_{m}, c_{m}\right),\left(c_{1}, b_{1}\right), \ldots,\left(c_{m}, b_{m}\right) \in \theta_{i}^{F(\mathbf{A}), K}, \\
& \exists \mathrm{p}_{1}, \mathbf{p}_{2}, \mathrm{q}_{1}, \mathrm{q}_{2} \in T_{\mathcal{F}}(\mathcal{X}) \text { such that } \mathrm{p}_{1} \approx \mathrm{p}_{2}, \mathbf{q}_{1} \approx \mathrm{q}_{2} \in \mathcal{E} \\
& \mathrm{p}_{1}^{F(\mathbf{A})}\left(a_{1}, \ldots, a_{m}\right)=a, \mathrm{q}_{1}^{F(\mathbf{A})}\left(b_{1}, \ldots, b_{m}\right)=b, \\
& \left.\mathrm{p}_{2}^{F(\mathbf{A})}\left(c_{1}, \ldots, c_{m}\right)=\mathrm{q}_{2}^{F(\mathbf{A})}\left(c_{1}, \ldots, c_{m}\right)\right\}
\end{aligned}
$$

Then

i) The homomorphism

$$
\operatorname{nat}_{\theta_{e}} \circ i: \mathbf{A} \rightarrow F(\mathbf{A}) / \hat{\theta}_{e}^{F(\mathbf{A}), K}
$$

is a $K$-reflection of $\mathbf{A}$.

ii) Let $\theta_{w}^{\mathbf{A}, M}=\theta_{e}^{F(\mathbf{A}), K} \cap A^{2}$. Then the natural homomorphism

$$
\operatorname{nat}_{\theta_{\mathrm{w}}}: \mathbf{A} \rightarrow \mathbf{A} / \theta_{\theta_{\mathrm{v}}^{\mathrm{A}}, M}
$$

is an $M$-refiection of $\mathbf{A}$.

\section{Tensor products}

Let us start by introducing the notions of bihomomorphism and tensor product

Definition 3. Given partial algebras $\mathbf{A}=\left(A,\left(f^{\mathbf{A}}\right)_{f \in F}\right)$, $\mathrm{B}=\left(B,\left(f^{\mathrm{B}}\right)_{f \in F}\right)$ and $\mathrm{C}=\left(C,\left(f^{\mathrm{C}}\right)_{f \in F}\right)$ of some type $\mathcal{F}$, a bihomomorphism of $\mathrm{A} \times \mathrm{B}$ into $\mathrm{C}$ is a mapping $h: A \times B \rightarrow C$ such that

- for any $b \in B, h(-, b): A \rightarrow C$ is a homomorphism from $\mathbf{A}$ into $C$, and

- for any $a \in A, h(a,-): B \rightarrow C$ is a homomorphism frorn $\mathrm{B}$ into C. 
In other words,

a) If $f \in F$ with $\eta(f)=0$ and $\operatorname{dom} f^{\mathrm{A}} \neq \emptyset$ then $\operatorname{dom} f^{\mathrm{C}} \neq \emptyset$ too, and for any $b \in B$

$$
h\left(f^{\mathbf{A}}, b\right)=f^{\mathbf{C}}
$$

b) If $f \in F$ with $\eta(f)>0$ and $\left(a_{1}, \ldots, a_{\eta(f)}\right) \in \operatorname{dom} f^{\mathrm{A}}$ for some $f \in F$ then for any $b \in B$

$$
\left(h\left(a_{1}, b\right), \ldots, h\left(a_{\eta(f)}, b\right)\right) \in \operatorname{dom} f^{\mathrm{C}} .
$$

and in that case

$$
f^{\mathrm{C}}\left(h\left(a_{1}, b\right), \ldots, h\left(a_{\eta(f)}, b\right)\right)=h\left(f^{\mathrm{A}}\left(a_{1}, \ldots, a_{\eta(f)}\right), b\right)
$$

c) Similar statements hold interchanging the rôles of $\mathbf{B}$ and $\mathbf{A}$.

Definition 4. Let $\mathbf{A}$ and $\mathbf{B}$ be two similar partial algebras and let $M$ be a class of algebras of the same type. Assume that there exists a partial algebra $\mathbf{T}$ of the same type such that

(i) $\mathbf{T} \in M$

(ii) There exists a bihomonorphism $\pi: \mathbf{A} \times \mathbf{B} \rightarrow \mathbf{T}$

(iii) Given any bihomomorphism $h: \mathbf{A} \times \mathbf{B} \rightarrow \mathbf{C}$ into some $\mathbf{C} \in M$, there exists a unique homomorphism $\tilde{h}: \mathbf{T} \rightarrow \mathbf{C}$ such that $\tilde{h} \pi=h$

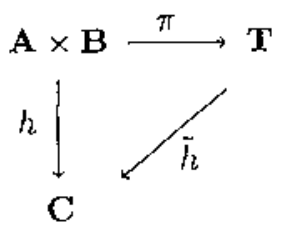

Then we shall call such a $T$ a tensor product of $A$ and $B$ w.r.t. $M$ (or $M$-tensor product of $\mathbf{A}$ and $\mathbf{B}$ ).

Notice that an algebra satisfying the properties above is unique up to isomorphism, so henceforth we shall talk about the $M$-tensor product of $\mathbf{A}$ and $\mathbf{B}$, and we shall denote it by $\mathbf{A} \otimes_{M} \mathbf{B}$. Moreover, we shall call the bihomomorphism $\pi$ the tensor bihomomorphism.

\section{Examples.}

1) If $\mathbf{A}$ and $\mathbf{B}$ are discrete algebras, then their tensor product w.r.t. the class of all partial alyebras of the same type is the discrete algebra over the cartesian product of their carriers.

2) If $M$ is a variety of total algebras and $\mathbf{A}, \mathbf{B} \in M$, then $\mathbf{A} \otimes_{M} \mathbf{B}$ can be taken as the teusor product on a variety as defined in [Fra]. 
3) Let $F=\left\{f_{0}, f_{1}\right\}$ with $\eta\left(f_{0}\right)=0$ and $\eta\left(f_{1}\right)=1$. Consider the partial algebras of type $(F, \eta) \mathbf{A}=\left(\left\{a_{1}, a_{2}\right\}, f_{0}^{\mathbf{A}}, f_{1}^{\mathbf{A}}\right)$ and $\mathbf{B}=\left(\left\{b_{1}, b_{2}\right\}, f_{0}^{\mathbf{B}}, f_{1}^{\mathbf{B}}\right)$ given by

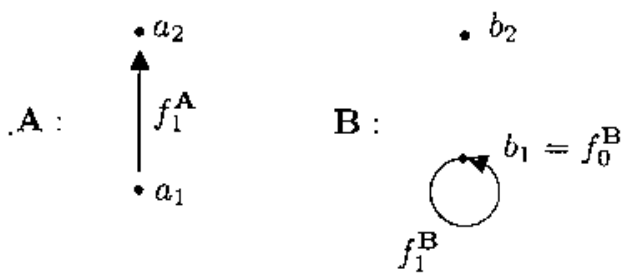

It is not difficult to check that the tersor product of $\mathbf{A}$ and $\mathbf{B}$ w.r.t. the class $M=\{\mathrm{A}, \mathrm{B}\}$ does not exist.

This last example shows that, in general, given arbitrary partial algebras $\mathbf{A}$ and $\mathbf{B}$ and class $M$, such $M$-tensor product need not exist, even if $\mathbf{A}, \mathbf{B} \in M$. Our main goal is to prove that if $M$ is quasi-primitive then $\mathbf{A} \otimes_{M} \mathbf{B}$ always exists.

Let $\mathbf{A}=\left(A,\left(f^{\mathbf{A}}\right)_{f \in F}\right)$ and $\mathbf{B}=\left(B,\left(f^{\mathrm{B}}\right)_{f \in F}\right)$ be two partial algebras of type $\mathcal{F}=(F, \eta)$. Let us consider a partial algebra $\overline{\mathbf{A} \times \mathbf{B}}=$ $\left(\overline{A \times B},\left(f^{\overline{\mathbf{A} \times \mathbf{B}}}\right)_{f \in F}\right)$ with carrier set

$$
\begin{gathered}
\overline{A \times B}=A \times B \cup\left\{f\left(\left(a_{1}, b\right), \ldots,\left(a_{\eta(f)}, b\right)\right) \mid f \in F,\right. \\
\left.\left(a_{1}, \ldots, a_{\eta(f)}\right) \in \operatorname{dom} f^{\mathrm{A}}, b \in B\right\} \\
\cup\left\{f\left(\left(a_{1} b_{1}\right), \ldots,\left(a, b_{\eta(f)}\right)\right) \mid f \in F ;\right. \\
\left.\left(b_{1}, \ldots, b_{\eta(f)}\right) \in \operatorname{dom} f^{\mathrm{B}}, a \in A\right\} \\
\cup\{f \mid f \in F, \eta(f)=0 \\
\text { and either dom } \left.f^{\mathrm{A}} \text { or dom } f^{\mathrm{B}} \text { are non-empty }\right\}
\end{gathered}
$$

(so, $\overline{A \times B}$ is a subset of the set $T_{\mathcal{F}}(A \times B)$ of terms of type $\mathcal{F}$ over $A \times B$ ) and operations $f^{\overline{\mathrm{A} \times \mathrm{B}}}, f \in F$, defined by

- If $\eta(f)=0$ then $\operatorname{dom} f^{\overline{\mathbf{A} \times \mathbf{B}}} \neq \emptyset$ iff either $\operatorname{dom} f^{\mathbf{A}}$ or $\operatorname{dom} f^{\mathbf{B}}$ are non-empty, and in that case $f^{\overline{\mathrm{A} \times \mathrm{B}}}=f \in \overline{A \times B}$

- If $\eta(f)>0$ then

$$
\begin{gathered}
\operatorname{dom} f^{\overline{\mathbf{A} \times \mathbf{B}}}=\left\{\left(\left(a_{1}, b\right), \ldots,\left(a_{\eta(f)}, b\right)\right) \mid\left(a_{1}, \ldots, a_{\eta(f)}\right) \in \operatorname{dom} f^{\mathbf{A}}, b \in B\right\} \\
\cup\left\{\left(\left(a, b_{1}\right), \ldots,\left(a, b_{\eta(f)}\right)\right) \mid\left(b_{1}, \ldots, b_{\eta(f)}\right) \in \operatorname{dom} f^{\mathbf{B}}, a \in A\right\}
\end{gathered}
$$

and if $\left(\left(a_{1}, b\right), \ldots,\left(a_{\eta(f)}, b\right)\right) \in \operatorname{dom} f^{\overline{\mathbf{A} \times \mathbf{B}}}$ then

$$
f^{\overline{\mathbf{A} \times \overline{\mathbf{B}}}}\left(\left(a_{1}, b\right), \ldots,\left(a_{\eta(f)}, b\right)\right)=f\left(\left(a_{1}, b\right), \ldots,\left(a_{\eta}(f) ; b\right)\right)
$$


while if $\left(\left(a, b_{1}\right), \ldots,\left(a, b_{\eta(f)}\right)\right) \in \operatorname{dom} f^{\overline{\mathrm{A} \times \mathbf{B}}}$ ther

$$
f^{\overline{\mathrm{A} \times \overline{\mathrm{B}}}}\left(\left(a, b_{1}\right), \ldots,\left(a, b_{\tau,}(f)\right)\right)=f\left(\left(a, b_{1}\right), \ldots,\left(a, b_{\eta(f)}\right)\right)
$$

In other words, $\overline{\mathbf{A} \times \mathbf{B}}$ is the relative subalgebra of the algebra $\mathbf{T}_{\mathcal{F}}(A \times$ $B$ ) of terms of type $\mathcal{F}$ over $A \times B$, the carrier set of which is the set $\overline{A \times B}$ defined above.

Now consider on $\overline{A \times B}$ the following relation

$$
\begin{aligned}
\theta_{0}= & \left\{\left(f\left(\left(a_{1}, b\right), \ldots,\left(a_{\eta(f)}, b\right)\right),\left(f^{\mathrm{A}}\left(a_{1}, \ldots, a_{\eta}(f)\right), b\right)\right) \mid\right. \\
& \left.\left.f \in F,\left(a_{1}, \ldots, a_{\eta(f)}\right) \in \operatorname{dom} f^{\mathrm{A}}, b \in B\right)\right\} \\
& \cup\left\{\left(f\left(\left(a, b_{1}\right), \ldots,\left(a, b_{\eta(f)}\right)\right),\left(a, f^{\mathrm{B}}\left(b_{1}, \ldots, b_{\eta(f)}\right)\right)\right) \mid\right. \\
& \left.\left.f \in F,\left(b_{1}, \ldots, b_{\eta(f)}\right) \in \operatorname{dom} f^{\mathrm{B}}, a \in A\right)\right\} \\
& \cup\left\{\left(\left(f^{\mathrm{A}}, b\right), f\right) \mid f \in F, \eta(f)=0, f^{\mathrm{A}} \in A, b \in B\right\} \\
& \cup\left\{\left(\left(a, f^{\mathrm{B}}\right), f\right) \mid f \in F, \eta(f)=0, f^{\mathrm{B}} \in B, a \in A\right\}
\end{aligned}
$$

and let $\theta$ be the least congruence on $\overline{\mathbf{A} \times \mathbf{B}}$ containing $0_{0}$. Set

$$
\mathbf{A} \otimes \mathbf{B}=\overline{\mathbf{A} \times \mathbf{B}} / 0
$$

Lemma 4. $\mathbf{A} \otimes \mathbf{B}$ is the tensor product of $\mathbf{A}$ and $\mathbf{B}$ w.r.t. the class of all partial algebras of type $\mathcal{F}$.

Proof: Let us check that such $\mathbf{A} \otimes \mathbf{B}$ satisfies conditions (i)-(iii) in the defirition of tensor product w.r.t. the class of all partial algebras of the given type.

(i) $\mathbf{A} \otimes \mathbf{B}$ is a partial algebra of type $\mathcal{F}$ by defirition.

(ii) The mapping $\pi: \mathbf{A} \times \mathbf{B} \rightarrow \mathbf{A} \otimes \mathbf{B}$ given by $\pi(a, b)=[(a, b)]$ (the equivalence class of $(a, b)$ with respect to $\theta)$ for any $a \in A, b \in B$ is a bihomomorphism.

Indeed, let $\left(a_{1}, \ldots, a_{\eta(f)}\right) \in \operatorname{dom} f^{\mathbf{A}}$ and $b \in B$. Then

$$
\left(\left(a_{1}, b\right), \ldots,\left(a_{T(f)}, b\right)\right) \in \operatorname{dom} f^{\overline{\mathrm{A} \times \mathrm{B}}}
$$

(by construction) and thus

$$
\left(\pi\left(a_{1}, b\right), \ldots, \pi\left(a_{\eta(f)}, b\right)\right)=\left(\left[\left(a_{1}, b\right)\right], \ldots,\left[\left(a_{\eta(f)}, b\right)\right]\right) \in \operatorname{dom} f^{\mathbf{A} \otimes \mathbf{B}}
$$


by the definition of the quotient algebra. Moreover,

$$
\begin{aligned}
\pi\left(f^{\mathbf{A}}\left(a_{1}, \ldots, a_{\eta(f)}\right), b\right) & =\left[\left(f^{\mathbf{A}}\left(a_{1}, \ldots, a_{\eta(f)}\right), b\right)\right]= \\
& =\left[f\left(\left(a_{1}, b\right), \ldots,\left(a_{\eta(f)}, b\right)\right)\right]= \\
& =\left[f^{\overline{\mathbf{A} \times \mathbf{B}}}\left(\left(a_{1}, b\right), \ldots,\left(a_{\eta(f)}, b\right)\right)\right]= \\
& =f^{\mathbf{A} \otimes \mathbf{B}}\left(\left[\left(a_{1}, b\right)\right], \ldots,\left[\left(a_{\eta(f)}, b\right)\right]\right)= \\
& =f^{\mathbf{A} \otimes \mathbf{B}}\left(\pi\left(a_{1}, b\right), \ldots, \pi\left(a_{\eta(f)}, b\right)\right)
\end{aligned}
$$

One proves in a similar way that if $\left(b_{1}, \ldots, b_{n(f)}\right) \in \operatorname{dom} f^{\mathrm{B}}$ and $a \in A$ then

$$
\left(\pi\left(a, b_{1}\right), \ldots, \pi\left(a, b_{\eta(f)}\right)\right) \in \operatorname{dom} f^{\mathbf{A} \otimes \mathbf{B}}
$$

and

$$
\pi\left(a, f^{\mathrm{B}}\left(b_{1}, \ldots, b_{\eta(f)}\right)\right)=f^{\mathbf{A} \otimes \mathbf{B}}\left(\pi\left(a, b_{1}\right), \ldots, \pi\left(a, b_{\eta(f)}\right)\right)
$$

To check the bihomomorphism condition for the 0-ary operations is straightforward.

(iii) Now, let $g: \mathbf{A} \times \mathbf{B} \rightarrow \mathbf{C}$ be any bihomomorphism. First of all, we see that it can be extended in a unique way to a homomorphism $\hat{g}: \overline{\mathbf{A} \times \mathbf{B}} \rightarrow \mathbf{C}$. Indeed, consider the mapping $\hat{g}: \bar{A} \times B \rightarrow C$ given by $\hat{g}(a, b)=g(a, b)$ if $a \in A, b \in B$

$$
\begin{aligned}
& \hat{g}\left(f\left(\left(a_{1}, b\right), \ldots,\left(a_{\eta(f)}, b\right)\right)\right)= g\left(f^{\mathbf{A}}\left(a_{1}, \ldots, a_{\eta(f)}\right), b\right) \\
& \text { if } f \in F,\left(a_{1}, \ldots, a_{\eta(f)}\right) \in \operatorname{dom} f^{\mathbf{A}}, b \in B \\
& \hat{g}\left(f\left(\left(a, b_{1}\right), \ldots,\left(a, b_{\eta(f)}\right)\right)\right)= g\left(a_{1} f^{\mathbf{B}}\left(b_{1}, \ldots, b_{\eta(f)}\right)\right) \\
& \text { if } f \in F,\left(b_{1}, \ldots, b_{\eta(f)}\right) \in \operatorname{dom} f^{\mathbf{B}}, a \in A \\
& \hat{g}(f)=f^{\mathbf{C}} \text { if } f \in F, \eta(f)=0, f \in \overline{A \times B}
\end{aligned}
$$

(Notice that, since $g$ is a bihomomotphism, if $f \in F,(\overbrace{a, \ldots, a}^{\eta(f)}) \in$ $\operatorname{dom} f^{\mathbf{A}}$ and $(\overbrace{b, \ldots, b}^{w(f)} \in \operatorname{dom} f^{\mathbf{B}}$ then $(g(a, b), \ldots, g(a, b)) \in \operatorname{dom} f^{\mathrm{C}}$ and

$$
g\left(f^{\mathrm{A}}(a, \ldots, a), b\right)=f^{\mathrm{C}}(g(a, b), \ldots, g(a, b))=g\left(a, f^{\mathrm{B}}(b, \ldots, b)\right)
$$

and that if $f$ is a 0 -ary operation symbol such that $f \in \overline{A \times B}$ then either $\operatorname{dom} f^{\mathrm{A}}$ or $\operatorname{dom} f^{\mathrm{B}}$ are non-emply, and it implies that $\operatorname{dom} f^{\mathrm{C}} \neq \emptyset$ too, again because $g$ is a bihomomorphism.) 
It is not difficult to prove that $\hat{g}$ is a homomorphism from $\overline{\mathbf{A} \times \mathbf{B}}$ into C. For instance, we check below the case of $\left(\left(a_{1}, b\right), \ldots,\left(a_{n(f)}, b\right)\right) \in$ $\operatorname{dom} f^{\overline{\mathrm{A} \times \mathrm{B}}}$, leaving the remaining ones to the reader.

If $\left(\left(a_{1}, b\right), \ldots,\left(a_{n(f)}, b\right)\right) \in \operatorname{dom} f^{\overline{\mathrm{A} \times \mathrm{B}}}$ Lhen $\left(a_{1}, \ldots, a_{\eta(f)}\right) \in \operatorname{dom} f^{\boldsymbol{\Lambda}}$ and thus, since $g$ is a bihomomorphism, $\left(g\left(a_{1}, b\right), \ldots, g\left(a_{\eta}(f), b\right)\right) \in \operatorname{dom} f^{C}$ and

$$
\begin{aligned}
\hat{g}\left(f^{\overline{\mathrm{A} \times \mathrm{B}}}\left(\left(a_{1}, b\right), \ldots,\left(a_{\eta(f)}, b\right)\right)\right) & =\hat{g}\left(f\left(\left(a_{1}, b\right), \ldots,\left(a_{\eta(f)}, b\right)\right)\right) \\
& =g\left(f^{\mathbf{A}}\left(a_{1}, \ldots, a_{\eta(f)}\right), b\right) \\
& =f^{\mathbf{C}}\left(g\left(a_{1}, b\right), \ldots, g\left(a_{\eta(f)}, b\right)\right) \\
& =f^{\mathbf{C}}\left(\hat{g}\left(a_{1}, b\right), \ldots, \hat{g}\left(a_{\eta(f)}, b\right)\right)
\end{aligned}
$$

It is also obvious that such a $\hat{g}$ extending $g$ is unique. Finally, it is straightforward from the definition that $\theta_{0} \subseteq$ ker $\hat{g}$. So it induces a unique homomorphism $\tilde{g}: \mathbf{A} \otimes \mathbf{B} \rightarrow \mathbf{C}$ which nakes the diagram

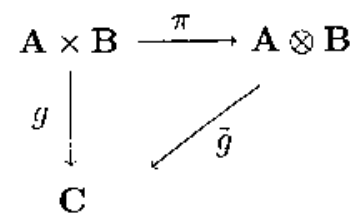

to commute.

This lemma allows us to prove that the tensor product w.r.t. quasiprimitive classes always exists, using Schmidt's theorem.

Theorem 5. Let $\mathbf{A}$ and $\mathbf{B}$ be two particl algebras of type $\mathcal{F}$ and let $M$ be a quasi-primitive class of partial algebras of type $\mathcal{F}$. Let $\mathbf{A} \otimes \mathbf{B}$ be defined as before and let $h: \mathbf{A} \otimes \mathbf{B} \rightarrow \mathbf{D}$ be an $M$-reftection of $\mathbf{A} \otimes \mathbf{B}$. Then $\mathbf{D}$ is the M-tensor product of $\mathbf{A}$ and $\mathbf{B}$.

Proof: Let $h: \mathbf{A} \otimes \mathbf{B} \rightarrow \mathbf{D}$ be an $M$-refection of $\mathbf{A} \otimes \mathbf{B}$. Ther on one hand $h \pi: \mathbf{A} \times \mathbf{B} \rightarrow \mathbf{D}$ is a bihomomorphism and $\mathbf{D} \in M$. So we only have to check that he satisfies the universal property (iii) in the definition of $M$-tensor product.

Let $g: \mathbf{A} \times \mathbf{B} \rightarrow \mathbf{C}$ be any bihomomorphism into an algebra $\mathbf{C} \in M$. By the previous lemma, there exists a unique homomorphism $g: \mathbf{A} \otimes \mathbf{B} \rightarrow$ C making commutative the following diagram 


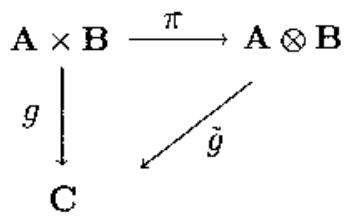

Now, by the universal property of $M$-reflections (see Theorem 1) there exists a unique $\bar{g}: \mathbf{D} \rightarrow \mathbf{C}$ making commutative the diagram
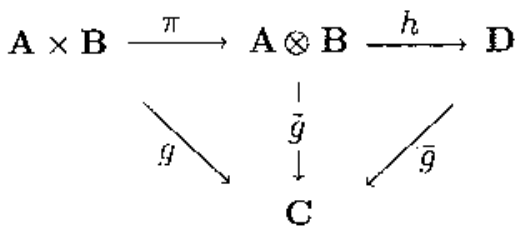

From this last diagram we get that there exists a homomorphism from $\mathbf{D}$ into $\mathbf{C}$, nanely $\bar{g}$; such that $g=\bar{g} h \pi$.

On the other side, it is not difficult to check (from the "uniqueness" of $\bar{g}$ given $g$, and of $\bar{g}$ given $\tilde{g}$ ) that $\bar{g}$ is the unique homomorphism with this property. This ends the proof.

Examples. Let $\mathbf{A}$ and $\mathbf{B}$ be two partial algebras of type $\mathcal{F}$, let $\mathbf{A} \otimes \mathbf{B}$ be as defined above and let $A \otimes B$ be the carrier set of $\mathbf{A} \otimes \mathbf{B}$.

1) The free completion $F(\mathbf{A} \otimes \mathbf{B})$ of $\mathbf{A} \otimes \mathbf{B}$ is the tensor product of $\mathbf{A}$ and $\mathbf{B}$ w.r.t. the class of all total algebras of type $\mathcal{F}$.

2) Let $K$ be a variely of total algebras of type $\mathcal{F}$. Let $\theta_{\mathrm{e}}^{F(\mathbf{A} \otimes \mathbf{B}), K}$ be the congruence on $F(\mathbf{A} \otimes \mathbf{B})$ introduced in Thm. 3. Then

$$
\mathbf{A} \otimes_{K} \mathbf{B} \cong F(\mathbf{A} \otimes \mathbf{B}) / \theta_{\theta}^{F(\mathbf{A} \otimes \mathbf{B}) \cdot K}
$$

by Thm. 3.(i).

3) Let $M=W(K)$, with $K$ as before, and let $\theta_{w}^{\mathbf{A} \otimes \mathbf{B}, M}=\theta_{e}^{F(\mathbf{A} \otimes \mathbf{B}), K} \cap$ $(A \otimes B)^{2}$. Ther

$$
\mathbf{A} \otimes_{W(K)} \mathbf{B} \cong \mathbf{A} \otimes \mathbf{B} / \theta_{\theta_{u}^{\mathrm{A}}}^{\mathrm{A} \otimes \mathbf{B}, M}
$$

by Thm. 3.(ii).

4) Let $M=E(K)$, with $K$ as before, and let $\theta_{e}^{\mathbf{A} \otimes \mathbf{B}, M}$ be the congruence on $\mathbf{A} \otimes \mathbf{B}$ introduced in Thm. 2. Then

$$
\mathbf{A} \otimes_{E(K)} \mathbf{B} \cong \mathbf{A} \otimes \mathbf{B} / \theta_{\epsilon}^{\wedge \otimes \mathbf{B}, M}
$$


by Thm. 2 .

5) More in general, Prop. 4 in [BMRRT] implies that if $M$ is a weakly guasi-primitive class of partial algebras ther $\mathbf{A} \otimes_{M} \mathbf{B}$ is isomorphic to the quotient of $\mathbf{A} \otimes \mathbf{B}$ by the least congruence on it such that the quotient belongs to $M$.

Let now $\mathrm{A}, \mathrm{B}$ be two partial algebras of type $\mathcal{F}$ and let $M_{1} \subseteq M_{2}$ be two quasi-primitive classes of partial algebras of the sarne type. Since $\mathbf{A} \otimes_{M_{1}} \mathbf{B} \in M_{1} \subseteq M_{2}$, there exists a (unique) homomorphism $h: \mathbf{A} \otimes_{M_{2}}$ $\mathbf{B} \rightarrow \mathbf{A} \otimes_{M_{1}} \mathbf{B}$ making the following diagram

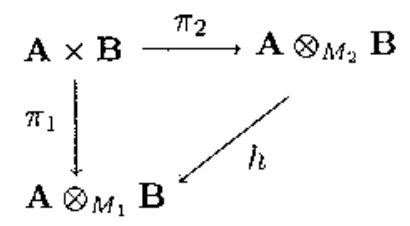

to commute (where $\pi_{1}$ and $\pi_{2}$ denote the corresponding tensor bihomomorphisms).

Proposition 6. With these notations, $h: \mathbf{A} \otimes_{M_{2}} \mathbf{B} \rightarrow \mathbf{A} \otimes_{M_{1}} \mathbf{B}$ is ant $M_{1}$-reflection.

Proof: Let $\mathbf{C} \in M_{1}$ and let $g: \mathbf{A} \otimes_{M_{2}} \mathbf{B} \rightarrow \mathbf{C}$ be a homomorphism. Then $g \pi_{2}: \mathbf{A} \times \mathbf{B} \rightarrow \mathbf{C}$ is a bihomomorphism, and therefore there exists a unique homomorphism $\bar{g}: \mathbf{A} \otimes_{M}, \mathbf{B} \rightarrow \mathbf{C}$ which rnakes the following diagram to commute

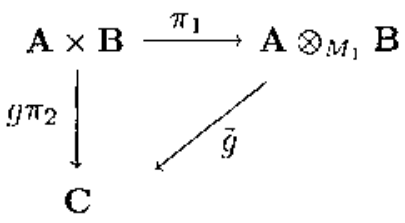

Since $\pi_{1}=h \pi_{2}$, we get that both $g$ and $\tilde{g} h$ make commutative the diagram 


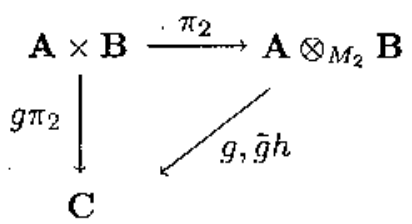

and thus, since $\mathrm{C} \in M_{1} \subseteq M_{2}$, by the universal property of the tensor product w.r.t. $M_{2}$ we get $g=\tilde{g} h$

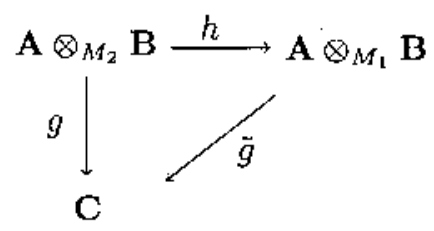

On the other hand, it is not difficult to prove that such a $\tilde{g}$ with $g=\tilde{g} h$ has to be unique. This shows that $h: \mathbf{A} \otimes_{M_{2}} \mathbf{B} \rightarrow \mathbf{A} \otimes_{M_{1}} \mathbf{B}$ satisfies the conditions of $M_{1}$-reflections.

Remark. This result generalizes Thm. 3 in [Gal] to the partial casc. A similar result was also proved in [Gri] taking $\mathbf{A}$ and $\mathbf{B}$ commutative semigroups, $M_{2}$ the variety of all semigroups and $M_{1}$ the subvariety of all commutative sernigroups.

As a particular case of the last Proposition, if $K$ is a variety then the inclusion

$$
W(K) \subseteq E(K)
$$

implies that there are natural mappings

$$
\mathbf{A} \otimes \mathbf{B} \rightarrow \mathbf{A} \otimes_{E(K)} \mathbf{B} \rightarrow \mathbf{A} \otimes_{W(K)} \mathbf{B}
$$

which are in fact reflections w.r.t. the corresponding classes. In general, all these tensor products are different (i.e. non-isomorphic), even for $\mathbf{A}, \mathbf{B} \in W(K)$, as the following example shows.

Example. Jet $F=\left\{f_{n} \mid n \in \mathbb{Z}^{+}\right\}$and $n\left(f_{n}\right)=1$ for all $n$. Let $K$ be the variety of all total algebras of type $(F, \eta)$ satisfying all equations $f_{n_{3}}\left(\ldots\left(f_{n_{p}}(x)\right) \ldots\right) \approx f_{\pi n_{1}}\left(\ldots\left(f_{n_{q}}(x)\right) \ldots\right), n_{1}+\cdots+n_{p}=m_{1}+\cdots+m_{q}$ Let $E(K)$ and $W(K)$ be the equational class and the weak subalgebras class of $K$. It turns out that $E(K) \neq W(K)$. (Actually, the class $E(K)$ 
can be identified with the class of all monounary ternal algebrus, and $W(K)$ with the class of all consistent monounary termal algebras; see for instance $[\mathbf{B R}]$, where in particular it is showed that these two classes are different.)

Let $\mathbf{A}, \mathbf{B} \in W(K)$ be the $(F, \eta)$-partial algebras given by the diagram
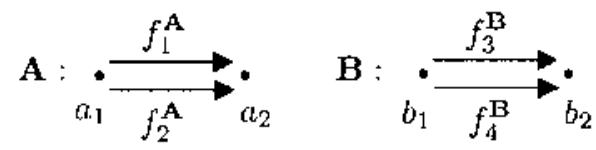

Notice that both $\mathbf{A}, \mathbf{B} \in W(K)$.

Applying the "recipes" in the examples after Thrn. 5 it is not difficult to prove that

a) $\mathbf{A} \otimes \mathbf{B}$ is given by the diagram (where, for simplicity, we skip the operations' superscripts and we use a single arrow to denote several operations if they give the same resuli)

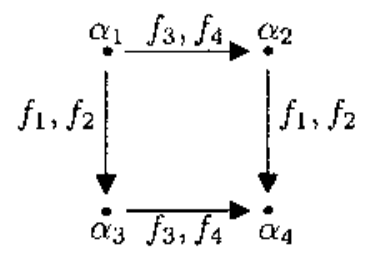

with the tensor homomorphism $\pi: \mathbf{A} \times \mathbf{B} \rightarrow \mathbf{A} \otimes \mathbf{B}$ given by

$$
\pi\left(a_{1}, b_{1}\right)=\alpha_{1}, \pi\left(a_{1}, b_{2}\right)=\alpha_{2}, \pi\left(a_{2}, b_{1}\right)=\alpha_{3}, \pi\left(a_{2}, b_{2}\right)=\alpha_{4} .
$$

Notice that $\mathbf{A} \otimes \mathbf{B} \notin E(K)$.

b) $\mathbf{A} \otimes_{E(K)} \mathbf{B}$ is given by the diagram

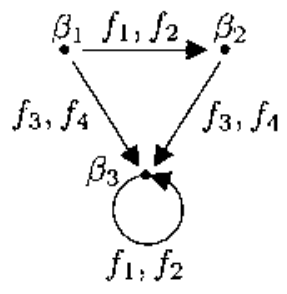

with the tensor hornomorphism $\pi: \mathbf{A} \times \mathbf{B} \rightarrow \mathbf{A} \otimes_{E(K)} \mathbf{B}$ given by

$$
\pi\left(a_{1}, b_{1}\right)=\beta_{1}, \pi\left(a_{1}, b_{2}\right)=\beta_{2}, \pi\left(a_{2}, b_{1}\right)=\pi\left(a_{2}, b_{2}\right)=\beta_{3} .
$$

It is not difficult to check that $\mathbf{A} \otimes_{E(K)} \mathbf{B} \notin W(K)$. 
c) $\mathbf{A} \otimes_{W(K)} \mathbf{B}$ is given by the diagram

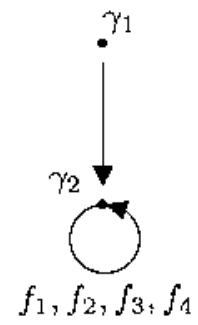

with the tensor homomorphism $\pi: \mathbf{A} \times \mathbf{B} \rightarrow \mathbf{A} \otimes_{W(K)} \mathbf{B}$ given by

$$
\pi\left(a_{1}, b_{1}\right)=\gamma_{1}, \pi\left(a_{2}, b_{1}\right)=\pi\left(a_{1}, b_{2}\right)=\pi\left(a_{2}, b_{2}\right)=\gamma_{3}
$$

In particular, from this example (and with the notations of $[\mathbf{B R}]$ ) we deduce that the tensor product of consistent termal algebras w.r.t. the class of termal algebras of the sane type need not be consistent.

\section{References}

[BMRR'i] BarTol, W., Monserrat, M., Rosserló, F., Rudak, L. AND Torrens, J., A note on least congruences related to termal algebras, Preprint, (1991).

[BR] Bartol, W. and Rosselló, F., Termal algebras: a guided tour, Preprint (1991).

[Bur] Burmeister, P.: "A model-theoretic approach to partial algebras," Mathematical Research Band 32, Akademie-Verlag, Berlin, 1986.

[BuS] Burris, S. and Sankappanavar, H., "A Course in Universal Algebra," Grad. Texts in Math. 78, Springer-Verlag, 1981.

[Fra] FRASER, G. A., Tensor product of semilattices and distributive lattices, Semigroup Forum 13 (1976), 178-184.

[Gal] Galanová, J., The tensor product, on at variely, in "Universal and Applied Algebra; Proc. of the $V$ Universal Algebra Symp., Turawa 1988:" (H. Hałkowska, B. Stawski eds.), World Scientific: 1989, pp. 92-98.

[Gri] GriLlet, P. A., The tensor product of commutative semigroups: Trans. Am. Math. Soc. 138 (1969), 28I-293.

[Ru] RUDAK, L., A completeness theorem for weak equational logic; Alg. Univ. 16 (1983), 331-337. 
[Sch] SCHMidr, J., A general existence theorem on partial algebras and its special cases, Colloq. Math. 14 (1966), 73-87.

Departanch de Ciències Matemàtiques i Informàtica Universilat de les Illes Balears 07071 Palma de Mallorea SPAIN

Rebut el 7 de Gener de 1992 